\title{
COMPARATIVE STUDY ON THE DYNAMIC RESPONSE OF RC 3-D FRAMES WITH AND WITHOUT VISCOUS DAMPERS
}

\author{
Aishwarya.M.M', Shilpa.B.R ${ }^{2}$ \\ ${ }^{1} P G$ Student, Department of Civil Engineering, Sambhram Institute of Technology, Bengaluru, Karnataka, India. \\ ${ }^{2}$ Assistant Professor, Department of Civil Engineering, Sambhram Institute of Technology, Bengaluru, Karnataka, \\ India.
}

\begin{abstract}
A structure can be called as earthquake safe if it is able to oppose the dynamic energy prompted by ground movement. It is impractical to make structure seismic safe all by its properties, for example, damping, stiffness and mass. In such cases we might have to give some outer device which either can oppose the force or ingest the dynamic power. Dampers, Bracings, Isolators are some viable gadgets which are the best reasonable instruments to moderate the quake powers.

In the present work an endeavor is made to minimize the dynamic reaction of the building by the establishment of inclining dampers. Response spectrum analysis is performed for $G+5, G+10$ and $G+15$ story 3-D structures for all the Indian seismic zones and medium soil condition. Dampers are installed in different patterns of mid-storeys(DMSD), alternative storeys(DTAS), and top five storeys(DTFS). Natural time period of structure, displacement, inter-storey drift ratio and accelerations are obtained. A comparative study is done on the models with and without viscous dampers(WOD) using SAP v 2000 software.
\end{abstract}

Keywords: Viscous Dampers, Modal Analysis, Response spectrum Analysis

\section{INTRODUCTION}

Together with traditional basic configuration it is vital to outline a building to with stand seismic loads. While outlining a structure for performance, durability and stability it is likewise important to make the move towards dissipation of energy and control of vibration. In the course of dynamic loading, structures may achieve the condition of resonance, which causes transmission of seismic power into the structure and subsequently to vibrate.

Absence of design of seismic resistance will bring about the loss of materials and lives. Repair of such harm after an earth tremor is normally costly and regularly requires clearing of the building.

\subsection{Modal Analysis}

The natural time period of structure is determined by performing Modal analysis. Also the time period is calculated as per to IS 1893, results obtained from both will be tabulated and compared. Code IS 1893: 2002(part-I) uses empirical formula to calculate natural time period which is straightly dependent on the plan dimension and building height while the analytical procedure calculates the natural period on the basis of mass and stiffness of the building.

\subsection{Non-Linear Static Analysis}

The current study Non-Linear static analysis is performed to obtain damper properties i.e, Mass and stiffness which are used to find out damping co-efficient (C). Using empirical formulae damping co-efficient is calculated. For RCC structures, damping ratio is taken as 0.05 . Using the empirical formulae damping co-efficient is obtained for the FEM analysis.

\subsection{Response Spectrum Analysis}

According to IS 1893:2002 PART I this analysis is carried out for both the directions $\mathrm{X}$ and $\mathrm{Y}$ and for all the earthquake zones in India to obtain the natural time period, acceleration, inter-storey drift, and displacement. Different soil conditions are present in the code, here the study of medium soil condition is considered.

\subsection{Objectives Of Study}

- Study on time period of structure before and after the addition of dampers.

- Comparison of time period obtained by modal analysis and IS 1893:2002.

- To find the lateral displacement, drift time-period and acceleration of structure in bare frame model and to compare it with the results obtained by other models equipped with dampers.

\section{SELECTION OF THE STRUCTURE}

1) Grid Data

a) Grid Spacing : Uniform $-6 \mathrm{~m}$ in both $\mathrm{X}$ and $\mathrm{Y}$ direction. 
b) Total Dimension: $24 \mathrm{~m} \times 24 \mathrm{~m}$

c) Grid Height : 3 meters

d) No. Of Stories : $(\mathrm{G}+5),(\mathrm{G}+10),(\mathrm{G}+15)$

e) Total Building Height: $18 \mathrm{~m}, 33 \mathrm{~m}, 48 \mathrm{~m}$

\section{2) Material Properties}

a) Grade of Concrete : M25

b) Grade of Steel: Fe - 500

c) Poisson's ratio: 0.2

\section{3) Frame Properties}

a) Type of frame :Special $\mathrm{RC}$ moment resisting frame fixed at the base

b) Size of beam : $(200 \times 500) \mathrm{mm}$

c) Size of column: $(500 \times 500) \mathrm{mm}$

g) Thickness of Slab : 150mm

\section{4) Static Loads}

a) Live load on floor: $3 \mathrm{KN} / \mathrm{m}^{2}$

b) Floor finish: $1.5 \mathrm{KN} / \mathrm{m}^{2}$

c) Wall load : $11.05 \mathrm{KN} / \mathrm{m}^{2}$

\section{5) Seismic Definition}

a) Earthquake Zone : II, III,IV,V

b) Damping ratio: $5 \%$

c) Importance factor: 1

d) Type of soil: Medium Soil

e) Respose Reduction Factor: 3

\section{MODELLING AND ANALYSIS OF BUILDING}

For the analysis work, 48 models are made. 16 models of $\mathrm{G}+5,16$ models of $\mathrm{G}+10$ and 16 models of $\mathrm{G}+15$ are modelled. DAMPERS are installed in different patterns of alternate storeys, mid storeys, top five storeys and analysed in SAPv2000.The parameters considered are lateral displacement, story drift, time period and acceleration.

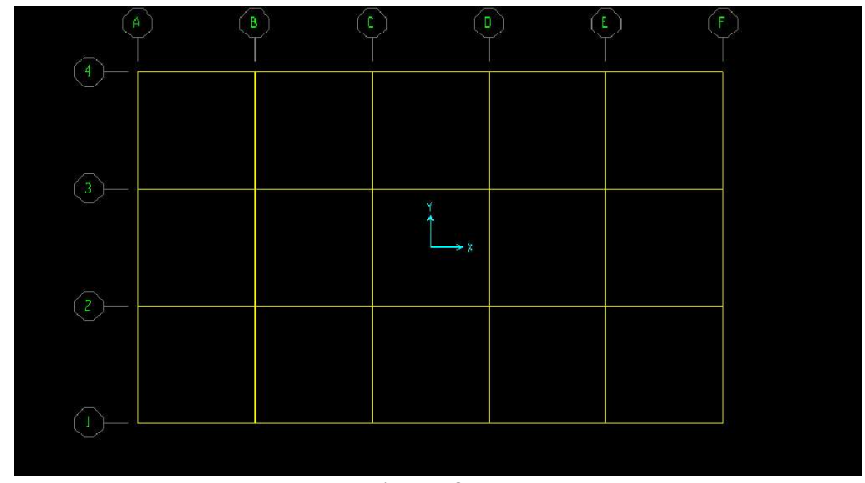

Fig.3.1.Plan of a structure

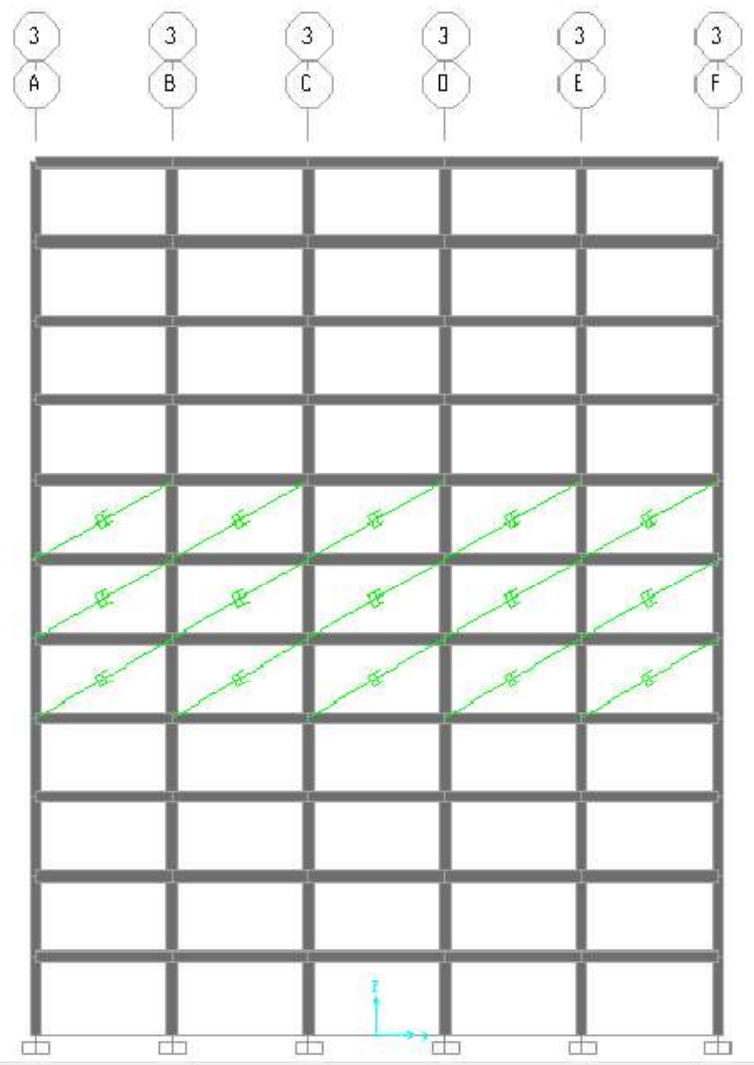

Fig.3.2. Elevation of $\mathrm{G}+10$ (with dampers at mid storeys) (DMSD) Structure
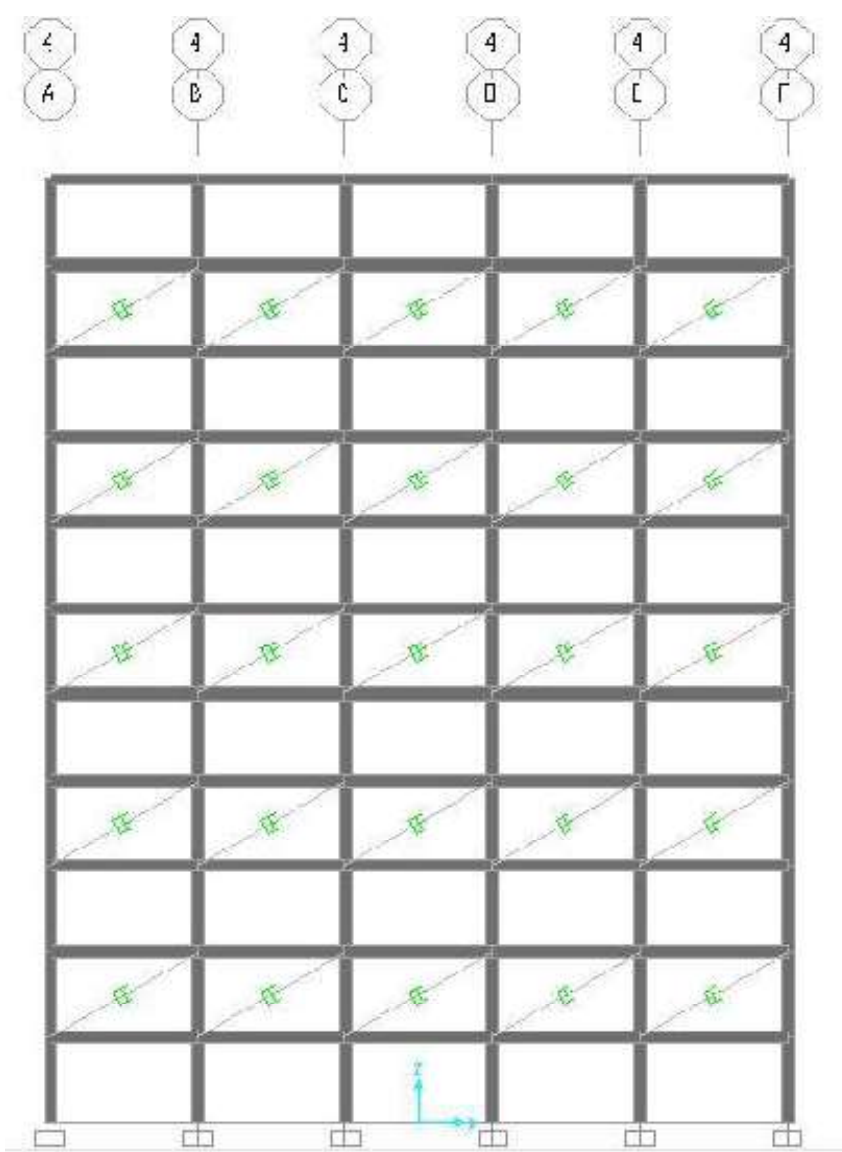

Fig.3.3. Elevation of $\mathrm{G}+10$ (with dampers at alternate storeys) (DTAS) Structure 


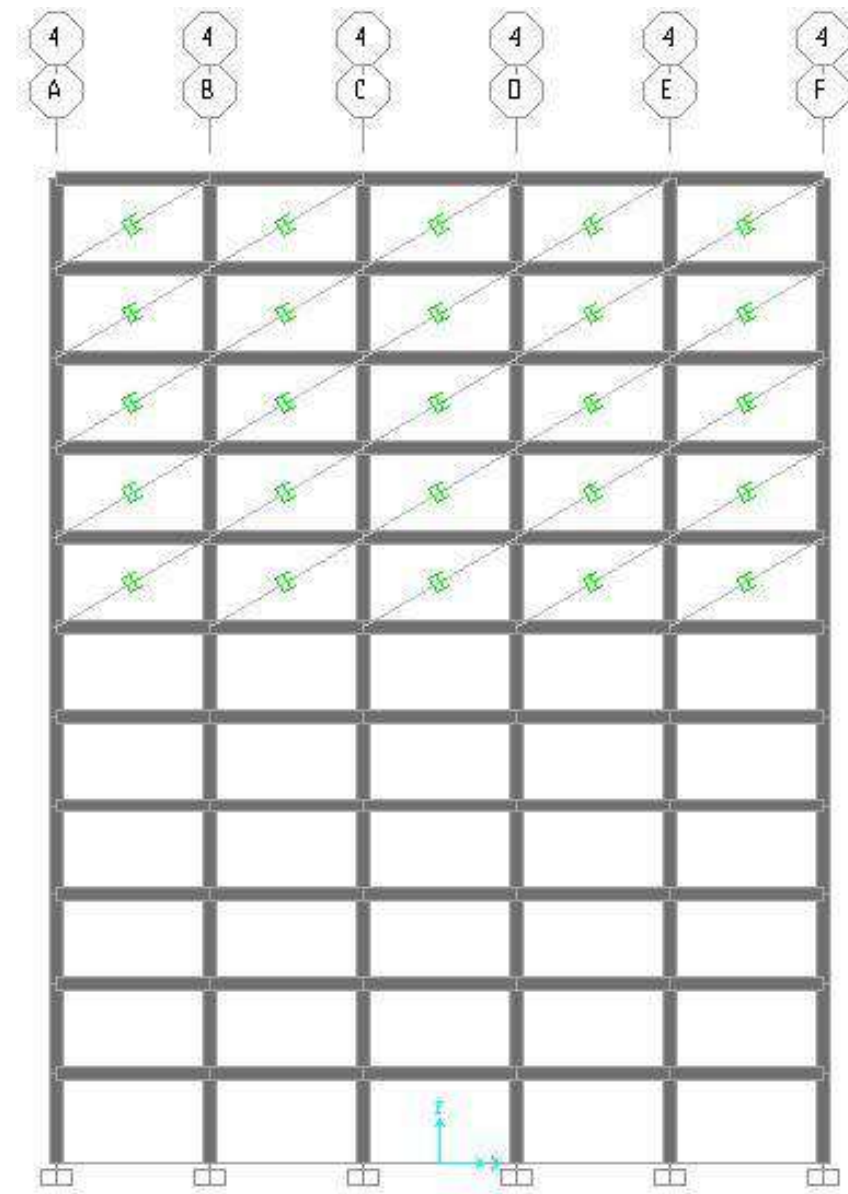

Fig.3.4. Elevation of $\mathrm{G}+10$ (with dampers at top five storeys) (DTFS) Structure

\section{RESULTS AND DISCUSSIONS}

\subsection{Time Period}

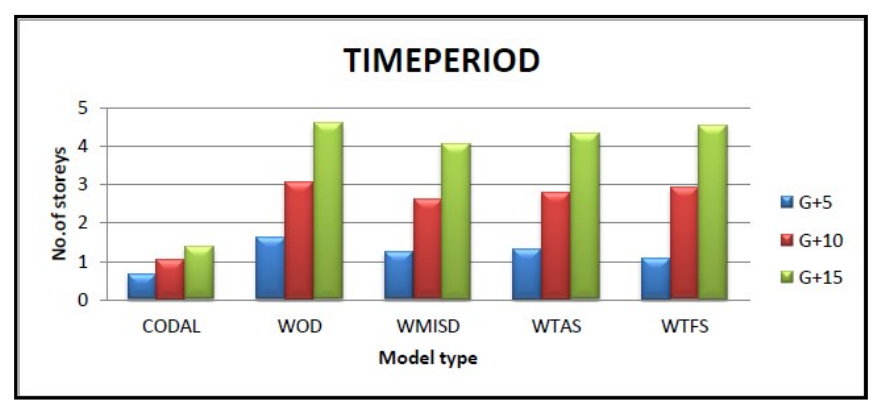

Fig 4.1 Time period for all the models from modal and codal analysis

Natural time period of the buildings increases as the number of storeys increases. Codal and modal analysis values show the similar effect of change in natural period due to change in height. Greater difference between the natural time period is obtained by codal method and modal analysis method. Only the height of building is considered in the code, but other parameters such as mass and stiffness are neglected. This indicates that height of the buildings is directly proportional to natural period with remaining parameters constant.

\subsection{Displacement}

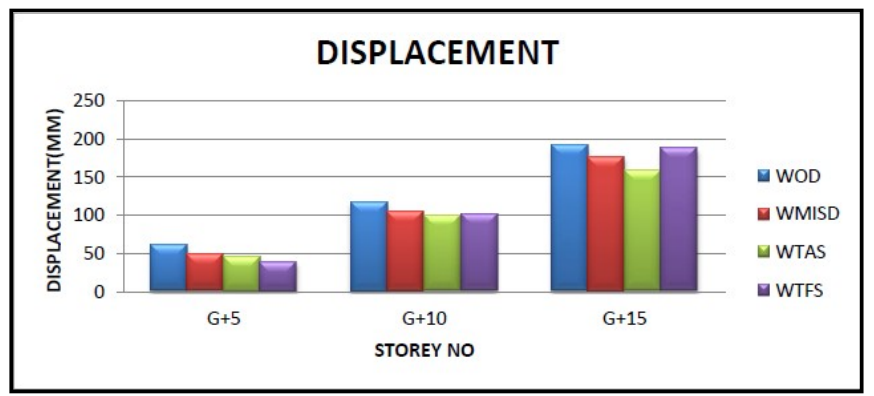

Fig 4.2: Displacement Vs Storey for all the models

For G+5 storey model, decreasing displacement is well observed as there is an increment in number of dampers. The model without damper has a roof displacement of $\mathbf{6 0 . 6 8}$ $\mathbf{m m}$ which reduces to $\mathbf{3 8 . 3 8} \mathbf{m m}$, almost $\mathbf{4 0 \%}$ reduction when dampers placed at top five storeys. For $\mathrm{G}+10$ storey model it is found that the effectiveness of damper is good when it is placed at alternative storeys. Displacement reduces from $117.31 \mathrm{~mm}$ to $\mathbf{9 9 . 0 1} \mathbf{~ m m}$, showing more than $15 \%$ reduction.

For $\mathrm{G}+15$ storey model it is notable that there is a decrease in displacement as there is an increment in number of dampers. Model without damper has a roof displacement of $192.00 \mathrm{~mm}$ which reduces to $158.31 \mathrm{~mm}$, almost $21 \%$ reduction when dampers placed at alternative storeys. Dampers are working most effectively when they are placed at alternative storeys and less effective when they are placed at storey with maximum inter-storey drifts.

\subsection{Drift}

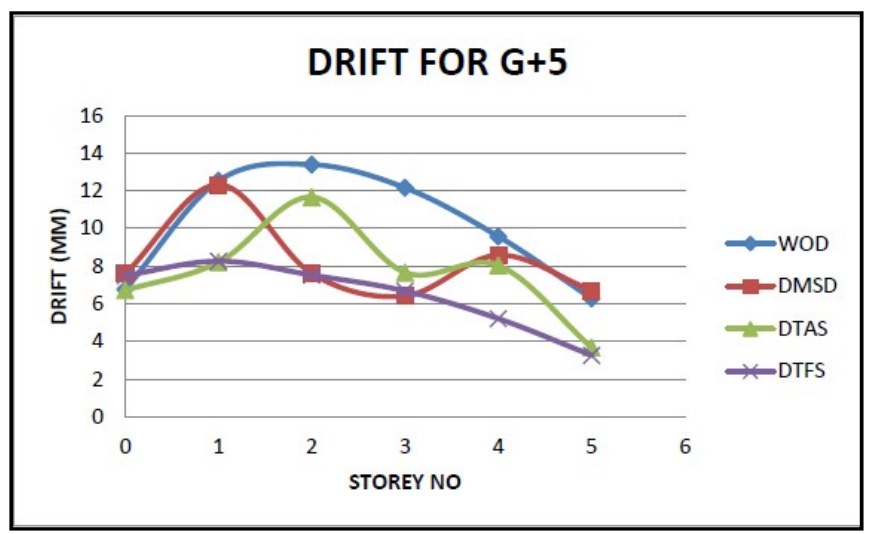

Fig 4.3.1: Drift Vs Storey for G+5 storey all the models

Up-to first storey drift reduces by a little value. Then reduction in drift is gradual. For model equipped with dampers to top five storey (DTFS) drift reduces in an effective manner as compared to DMSD and DTAS models. At the top storey drift is $\mathbf{6 . 2 7 2}$, for DTFS which is then reduced to $\mathbf{3 . 2 5 2}$ showing a reduction of more than $45 \%$. In between storeys effect is good.There is a reduction from $\mathbf{1 2 . 5 3 3}$ to $\mathbf{8 . 2 5 6}$ indicating more than $33 \%$ reduction wit DTAS. 


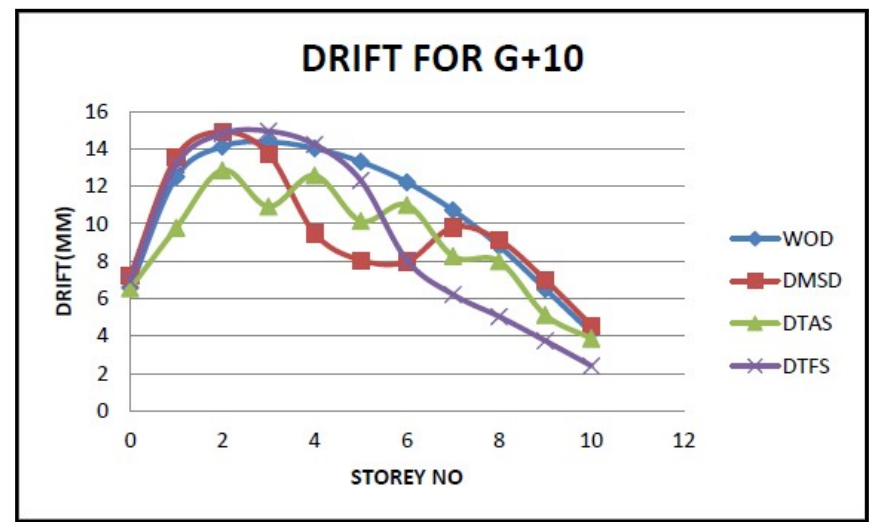

Fig 4.3.2: Drift Vs Storey for G+10 storey all the models

Up-to first storey drift reduces by a little value. Then reduction in drift is gradual. For model equipped with dampers to alternative storey (DTAS) drift reduces effectively as compared with the values of drift for DMSD and DTFS. DTAS model shows the zig-zag reduction pattern DMSD and DTFS models do not shows the complete reduction for drift At the first storey drift is $\mathbf{1 3 . 5 7 9}$ which is reduced to $\mathbf{9 . 7 7 8}$. At the top storey drift is $\mathbf{4 . 5 4 4}$ which is then reduced to $\mathbf{2 . 3 9 2}$ for DTFS indicating $45 \%$ reduction. Effect is very good in the between storeys where we can see a reduction up-to $\mathbf{5 0 \%}$.

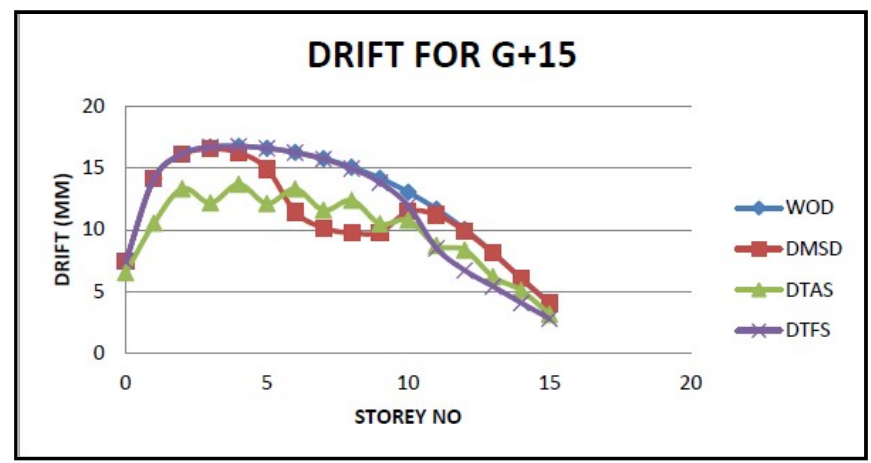

Fig 4.3.3: Drift Vs Storey for G+15 storey all the models

Up-to first storey drift reduces by a little value. Then reduction in drift is gradual. For model equipped with dampers to alternative (DTAS) drift reduces effectively as compared with the values of drift for DMSD and DTFS. Reduction in drift can be seen when dampers placed at alternative storeys .DTAS models showing positive result for all the storeys. DMSD and DTFS models do not shows the complete reduction for drift. DTAS model shows the zig-zag reduction pattern. Without damper(WOD) model which has maximum drift of $\mathbf{1 4 . 1 4 5}$ at 2 nd storey is reduced $\mathbf{1 0 . 5 3 4}$ more than $\mathbf{2 5 \%}$ reduction and $\mathbf{1 6 . 6 7}$ at $3 \mathrm{rd}$ storey is reduced to $\mathbf{1 2 . 1 6 6}$ more than $\mathbf{2 7 \%}$ reduction.

\subsection{Acceleration}

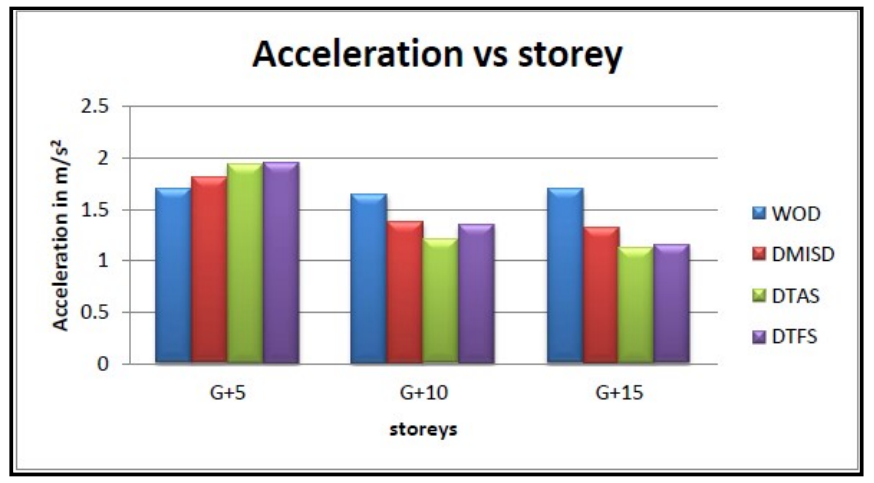

Fig 4.4: Acceleration Vs Storey all the models

For any structure, acceleration goes on decreasing as the height of the building increases. The acceleration of building is increased after addition of supplemental damping.

\section{CONCLUSION}

From the results it is found that supplemental damping required for the structure can be given by viscous dampers to reduce the dynamic response such as displacement and inter-storey drift. Among DMSD, DTAS and DTFS damper distribution pattern DTAS pattern found to be more effective to reduce the response in terms of displacement and drift for all the models. Results shows that uniform distribution of dampers within a building will absorb excess energy so that reduction in displacement and inter storey drift will take place.

\section{REFERENCES}

[1]. Afiya (2014) "Seismic Response of Multi-storey RC Buildings With And Without Viscous Dampers", DOSCE, UBDTCE, Davangere.

[2]. Abdelouahab Ras, Baghdad Boukhari , Nadir Boumechra and Karim Hamdaoui (2014) "Dissipative Capacity Analysis of Steel Building Using Viscous bracing Device" International Conference on Geological and Civil Engineering IPCBEE vol.62 (2014) (C) (2014) IACSIT Press, Singapore DOI: 10.7763/IPCBEE. 2014. V62. 4.

[3]. D.G. Weng, C. Zhanga, X.L. Lu, S. Zeng and S.M. Zhang (2012) " A simplified design procedure for seismic retrofit of earthquake-damaged RC frames with viscous dampers", Structural Engineering and Mechanics, Vol. 44, No. 5 (2012) 000-000.

[4]. Dhananjay A. Chikalekar, M.M. Murudi (2015) "Seismic performance of structure with fixed base, Base isolated structure and Structure with Viscous damper" ,International Journal of Research in Engineering \& Technology, Vol.4,Issue 9, eISSN: 2319-1163 | pISSN: 23217308.

[5]. Julious Marko (2006) "Influence of Damping On Building Systems Subjected to Seismic Effect", May 2006.

[6]. Bureau of Indian Standards: IS-1893, part 1 (2002), Criteria for Earthquake Resistant Design of Structures: Part 1 General provisions and Buildings, New Delhi, India.

[7]. Bureau of Indian Standards: IS-875, part 1 (1987), Dead Loads on Buildings and Structures, New Delhi, India. 